\title{
Cryo-Electron Tomography of Frozen-Hydrated Sections of Eukaryotic Cells
}

\author{
C. Schwartz, D. Nicastro, M.S. Ladinsky, D. Mastronarde, E. O’Toole and J.R. McIntosh \\ Boulder Lab for 3-D EM of Cells, Dept. MCD Biology, Univ. of Colorado, Boulder, CO 80309.
}

Electron tomography (ET) of chemically fixed, plastic embedded and sectioned specimens has become a useful method for studies of 3-D cell structure [1]. Fixation, embedding, and staining make biological samples comparatively resilient to the electron beam, which enables a routine application of ET to plastic sections. However, plastic embedded specimens can suffer artifacts from fixation, dehydration, sectioning, and section collapse during electron irradiation [2,3]. Consequently, there is great current interest in cryo-ET of unfixed and unstained frozen-hydrated samples, which allows visualization of cellular structures in a near-to-native state. To date, cryo-ET has been used primarily for specimens that can be embedded in vitreous ice by plunge freezing, such as macromolecular complexes, isolated organelles and small bacterial cells, because the sample thickness that can be imaged by cryo-EM is limited to $\sim 0.5 \square \mathrm{m}$ [4]. Thus cryo-ET of eukaryotic cells has been restricted to very thin regions of the cell like lamellipodia or flagella $[5,6]$. Here we show that cryo-microtomy permits cryo-ET of samples like yeasts and mitotic mammalian cells that are thick enough to require high-pressure freezing for vitrification.

We are using high-pressure frozen fission yeast as a model system with which to evaluate the quality of tomograms generated from sections. Log phase yeasts were frozen in interlocking brass hats with shallow, flat bottoms and domed, lecithin-coated tops [7]. After mounting the cell pellets directly in the cryo-microtome using a customized pin, $150-200 \mathrm{~nm}$ sections were cut at $-160^{\circ} \mathrm{C}$ with a $35^{\circ}$ cryo-diamond knife and collected on oyster grids that were coated with a supporting carbon film. Single-axis tilt series over $+/-60^{\circ}$ with $1.5^{\circ}$ increments were acquired as digital images (Fig 1a), using a Tecnai F-30 and a locally written microscope control program, SerialEM. Total dose to the specimen was $<100 \mathrm{e}^{-} / \AA^{2}$. Individual tilt series images were then aligned, reconstructed by weighted backprojection and analyzed using the IMOD software package [8].

The tomographic slice in Figure $1 \mathrm{~b}$ shows a cross section through the mitotic spindle midzone between two daughter nuclei of a fission yeast cell during anaphase B. Many structural details are clearly visible in the reconstruction: the nuclear envelope forms a narrow tube around a bundle of spindle microtubules; endoplasmatic reticulum closely approaches the nuclear envelope (Figs. $1 b, c, d)$. The microtubule bundle in this region consists of six interdigitating microtubules, 3 of which terminate in the reconstructed volume (Fig. 1d). The microtubules are square-packed with a mean center-center spacing of $40 \mathrm{~nm}$. Many neighbors are connected by bridges (Fig. 1b-inset). The reliability of frozen-hydrated specimens is shown in the $25 \mathrm{~nm}$ diameter of these microtubules, which confirms studies on native polymers in vitro.

Cryo-microtomy is a challenging technique; artifacts like crevasses (Fig. 1a) and section compression are common [9]. However, the tomographic reconstructions show that the crevasses are of minor importance for cryo-ET as they are confined to one side of the section and a third of the section thickness, leaving two-thirds of the volume undisturbed. Our results confirm that electron tomography can successfully be extended to cells and tissues using frozen-hydrated sections [10]. This opens up a wide field of biological problems that will benefit from this imaging technique that is uniquely suited to reveal the 3-D organization of complex and pleomorphic structures with macromolecular resolution. 


\section{References}

[1] J.R. McIntosh, J. Cell Biol. 153 (2001) 25-32. [2] R.A. Steinbrecht and M. Mueller, Cryotechniques in Biological Electron Microscopy, Springer-Verlag, Berlin, 1987. [3] P.K. Luther et al., Ultramicroscopy 24 (1988) 7-18. [4] W. Baumeister, Curr. Opin. Struct. Biol. 12 (2002) 67984. [5] O. Medalia et al., Science 298 (2002) 1209-13. [6] D. Nicastro, Proceedings ICEM-15, Durban, SA, 3 (2002) 209. [7] S. Craig et al., J. Microsc. 148 (1987) 103-6. [8] J.R. Kremer et al., J. Struct. Biol. 116 (1996) 71-6. [9] N. Sartori et al., J. Microsc. 192 (1998) 194. [10] C.-E. Hsieh et al., J. Struct. Biol. 138 (2002) 63-73. [11] This research was supported by NIH Biotechnology Resources Grant \#RR00592.
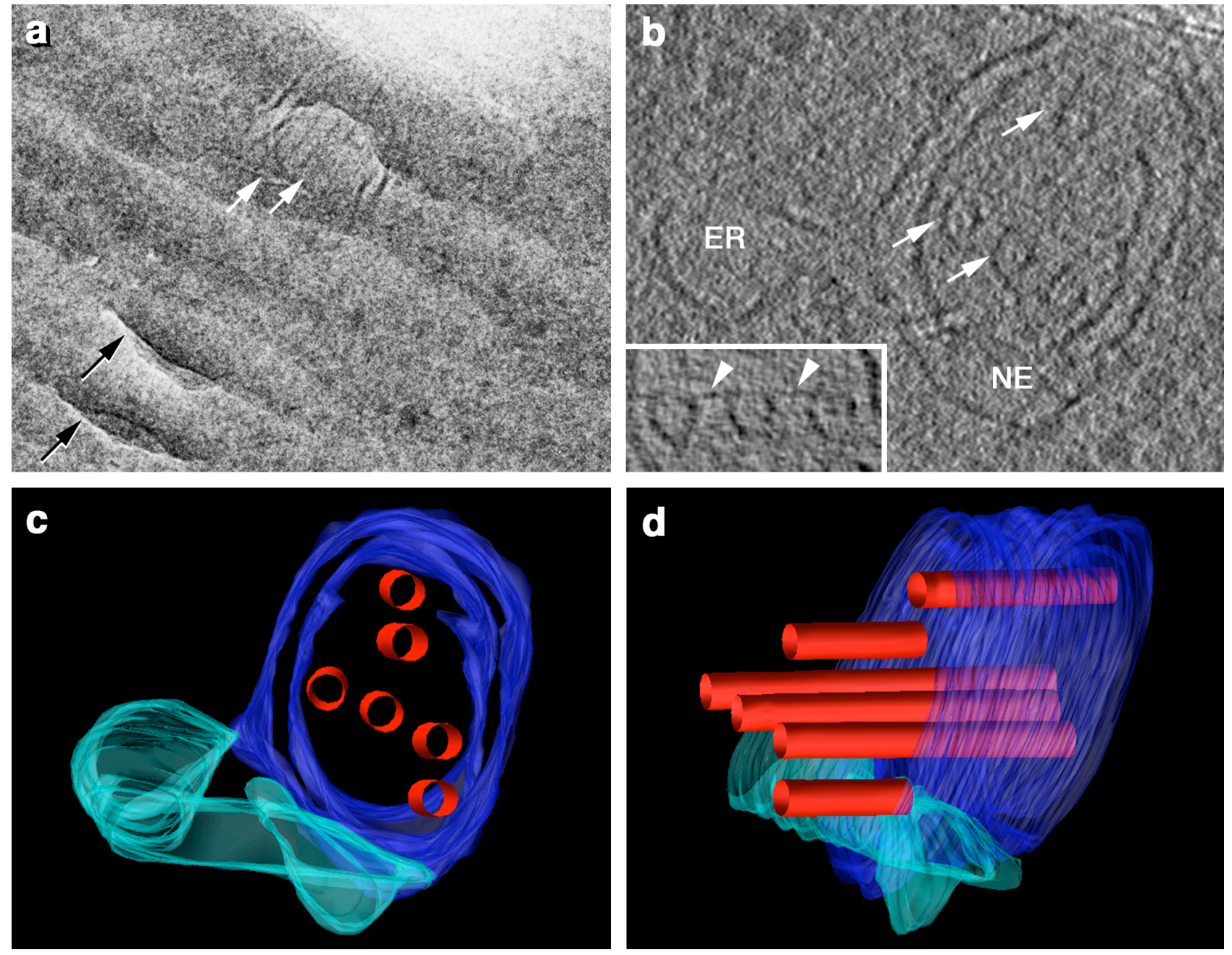

FIG. 1. Images from an original tilt series and from a tomographic reconstruction of a mitotic fission yeast cell. (a) Zero-degree projection from the original tilt series containing spindle microtubules (white arrows); note the crevasses perpendicular to the cutting direction (black arrows). (b) $10 \mathrm{~nm}$ thick tomographic slice showing a cross-section through the spindle microtubules (white arrows) and the surrounding nuclear envelope $(N E)$; on one side endoplasmic reticulum $(E R)$ closely approaches the outer nuclear membrane. (b-inset) $20 \mathrm{~nm}$ thick tomographic slice showing the bridges (white arrowheads) connecting adjacent microtubules. (c,d) Surface-rendering representations of the modeled spindle midzone showing the nuclear envelope (blue), spindle microtubules (red) and endoplasmic reticulum (cyan). Bars: $100 \mathrm{~nm}$ (a), $50 \mathrm{~nm}$ (b), $25 \mathrm{~nm}$ (b-inset). 